\title{
QUARTIC SURFACES OF ELLIPTIC RULED TYPE
}

BY

\author{
YUMIKO UMEZU
}

\begin{abstract}
Let $X$ be a normal quartic surface whose resolutions are birationally equivalent to elliptic ruled surfaces. We classify the singularities on $X$ and then describe the global structure of $X$.
\end{abstract}

0. Introduction. It is known [3] that the minimal resolution of a normal quartic surface in $\mathbf{P}^{3}$ over an algebraically closed field $k$ of characteristic $\neq 2,3$ is either (i) a $K 3$ surface, (ii) a rational surface, (iii) birationally equivalent to an elliptic ruled surface, or (iv) a ruled surface of genus 3. We shall investigate the structure of normal quartic surfaces of type (iii). In the sequel we call such a surface simply a quartic surface of elliptic ruled type.

Let $X$ be a quartic surface of elliptic ruled type and let $\pi: \tilde{X} \rightarrow X$ denote the minimal resolution of $X$. We shall study $X$ via the structure of $\tilde{X}$ and a linear system on $\tilde{X}$ which defines the morphism $\pi$.

Since the dualizing sheaf $\omega_{X}$ of $X$ is trivial, we can apply the results in [3]. Here we restate some of them (restricting to our present situation) which will play essential roles throughout this research. We use the terms and facts cited in $\S 1$ of [3] without notice.

LeMma 1. For any point $P$ on $X$, the geometric genus $p_{g}(P)$ of $P$ is not greater than 2.

Since $\omega_{X} \cong O_{X}$, there exists a unique effective anticanonical divisor on $\tilde{X}$ whose connected components correspond by $\pi$ to singular points with $p_{g} \geqslant 1$ on $X$. We denote this divisor by $\tilde{D}$.

LEMMA 2. Let $\tilde{X}=X_{n} \stackrel{\mu_{n}}{\rightarrow} X_{n-1} \stackrel{\mu_{n-1}}{\rightarrow} \cdots \stackrel{\mu_{1}}{\rightarrow} X_{0}=\bar{X}$ be a sequence of blow-downs obtaining a relatively minimal model $\bar{X}$ of $\tilde{X}$, and let $\tilde{H}$ be the pull-back of a general hyperplane section of $X$ to $\tilde{X}$ such that $\tilde{H}$ is irreducible and nonsingular. Put

$$
\begin{array}{llll}
H_{n}=\tilde{H}, & H_{i}=\mu_{i+1} \circ \mu_{i+2} \circ \cdots \circ \mu_{n}\left(H_{n}\right) & (0 \leqslant i \leqslant n-1), & \bar{H}=H_{0}, \\
D_{n}=\tilde{D}, & D_{i}=\mu_{i+1} \circ \mu_{i+2} \circ \cdots \circ \mu_{n}\left(D_{n}\right) & (0 \leqslant i \leqslant n-1), & \bar{D}=D_{0} .
\end{array}
$$

Then we have:

(i) $D_{i} \in\left|-K_{X_{i}}\right|(0 \leqslant i \leqslant n)$;

Received by the editors November 24, 1982 and, in revised form, March 4, 1983.

1980 Mathematics Subject Classification. Primary 14E15; Secondary 14J17.

Key words and phrases. Minimal resolution of singularities, exceptional set, geometric genus, elliptic ruled surfaces. 
(ii) $\mu_{i}$ is a blow-up with center at a point on $\operatorname{supp}\left(D_{i-1}\right) \cap \operatorname{supp}\left(H_{i-1}\right)$;

(iii) $D_{i}=\mu_{i}^{*}\left(D_{i-1}\right)-E_{i}$ where $E_{i}$ is the exceptional curve of the first kind for $\mu_{i}$ $(1 \leqslant i \leqslant n)$.

LEMma 3. On the elliptic ruled surface $\bar{X}^{\bar{\omega}} \rightarrow$, the effective anticanonical divisor $\bar{D}$ is one of the following types:

(i) $\bar{D}=C_{0}+C_{1}$, where $C_{0}$ is a minimal section of $\tilde{\omega}$ and $C_{1}$ is another section disjoint from $C_{0}$.

(ii) $\bar{D}=2 C_{0}+\sum f_{i}$, where $C_{0}$ is as above and the $f_{i}$ 's are fibres of $\tilde{\omega}$.

First, in $\S 1$, we make a list of possible singularities on $X$ with $p_{g} \geqslant 1$. After that, restricting ourselves to the case of characteristic 0 , we study the structure of $X$ in detail using the sequence of blow-ups as in Lemma 2.

We note that M. Kato and I. Naruki are studying the singularities on normal quartic surfaces to get similar results. However, their method is the analysis of homogeneous polynomials of degree 4 , which is quite different from ours.

Recently I received a manuscript from D. Epema, in reply to my letter enclosing my manuscript of the present paper, in which he also studies singular surfaces of the same kind.

1. Types of singularities on $X$. In this section we assume that $X$ is a normal quartic surface of elliptic ruled type, and we list the possibility of the types of singularities on $X$ with positive geometric genus. It will be shown later that every member in our list really appears as a singularity on a normal quartic surface of elliptic ruled type.

\section{LEMma 4. The multiplicity of each singular point on $X$ is equal to two.}

Proof. Let $P$ be a singular point on $X$, and let $p: X \rightarrow \mathbf{P}^{2}$ be the projection with center at $P$. If mult ${ }_{P} X=4$, then $X$ is a cone over a plane quartic curve, and hence the minimal resolution of $X$ is a ruled surface of genus 3 . If mult ${ }_{P} X=3$, then $p$ turns out to be a birational map onto $\mathbf{P}^{2}$, so $X$ is rational. Therefore we have mult $_{P} X=2$. Q.E.D.

By the lemmas in $\S 0$, the singularities on $X$ with $p_{g} \geqslant 1$ are either two simple elliptic singularities or a singularity with $p_{g}=2$. In what follows we use the notation of $\S 0$.

Proposition 1. If $X$ has two simple elliptic singularities, then they have the same type $\tilde{E}_{7}$ or $\tilde{E}_{8}$.

Proof. Let $P$ and $Q$ be the two simple elliptic singular points on $X$. We first show that the line $l$ through $P$ and $Q$ in $\mathbf{P}^{3}$ does not lie on $X$. Indeed, if $l$ is contained in $X$, let $\tilde{l}$ be the proper transform of $l$ on $\tilde{X}$. Then $\tilde{l} \cap \tilde{C}_{0} \neq \varnothing, \tilde{l} \cap \tilde{C}_{1} \neq \varnothing$ and $\tilde{l} \cdot \tilde{H}=1$, where $~$ means the proper transform in $\tilde{X}$. Since $\tilde{l}$ is isomorphic to $\mathbf{P}^{1}$, $\mu(\tilde{l})$ must be a fibre of $\bar{X}$ on which there is no center of the blow-ups $\mu: \tilde{X} \rightarrow \bar{X}$. So $\bar{H} \cdot f=1$ for any fibre $f$ of $\bar{X}$ and we get a contradiction because $\tilde{H}$ is a curve of genus 3. 
To prove the proposition, it is enough to show that if $P$ is of type $\tilde{E}_{7}$, then so is $Q$, since a simple elliptic singularity of multiplicity 2 is either of type $\tilde{E}_{7}$ or $\tilde{E}_{8}$. Consider the following commutative diagram:

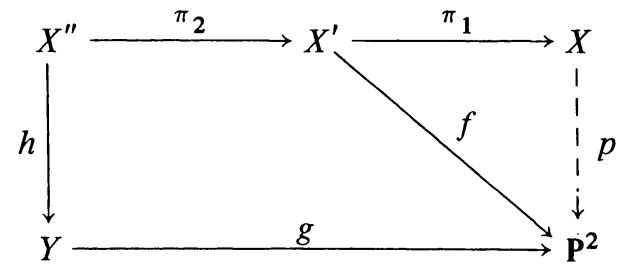

where $p$ is the projection from $X$ with center at $P, \pi_{1}: X^{\prime} \rightarrow X$ is the blow-up with center at $P, \pi_{2}: X^{\prime \prime} \rightarrow X^{\prime}$ is the normalization of $X^{\prime}$ and $g: Y \rightarrow \mathbf{P}^{2}$ is the finite morphism of degree 2 obtained by the Stein factorization of $f \circ \pi_{2}$. The degree of the branch locus of $f$ is equal to 6 , and $X^{\prime}$ is not normal since $P$ is of type $\tilde{E}_{7}$ (Laufer [2]). Therefore, the degree of the branch locus of $g$ is less than or equal to 4. Since $l \subsetneq X$, there is a neighbourhood $U$ of $Q$ in $X$ such that $U$ is transformed by $\pi_{1}^{-1}, \pi_{2}^{-1}$ and $h$ isomorphically into $Y$. So $Y$ has a simple elliptic singularity which is isomorphic to $Q$. Thus by the classification of plane curves of degree less than or equal to 4 (cf. Hidaka and Watanabe [1]), we prove that $Q$ is of type $\tilde{E}_{7}$. Q.E.D.

Proposition 2. Suppose $X$ has a singular point $P$ of geometric genus equal to two. Then the exceptional set for the minimal resolution of $P$ is one of the following four types:

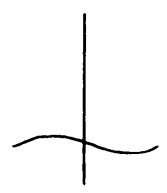

(i) $E 1+A_{1}$

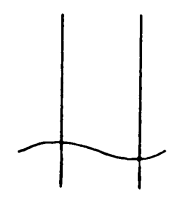

(ii) $E 1+A_{1}+A_{1}$

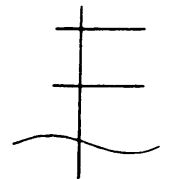

(iii) $E 1+D(3)$

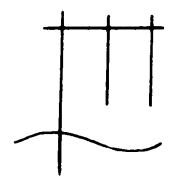

(iv) $E 1+D(4)$

where each curved line stands for a nonsingular elliptic curve whose self-intersection number is equal to -1 in (i), -2 in (ii)-(iv), and any straight line denotes a nonsingular rational curve with self-intersection number -2 .

(The symbols of these four types of singularities are due to S. S.-T Yau.)

Proof. Since $p_{g}(P)=2$, and $P$ is a Gorenstein singularity, we have $p_{a}(P)=1$ (Hidaka and Watanabe [1]). So we can define the elliptic sequence $Z_{B_{0}}, \ldots, Z_{B_{l+1}}$ according to Yau [4]. By definition, every $Z_{B_{i}}$ is an effective divisor supported on the exceptional set $\operatorname{supp}(\tilde{D})$ on $\tilde{X}$. By Lemmas 2 and $3, \operatorname{supp}(\tilde{D})$ is with simple normal crossings and has nonsingular elliptic curve $\tilde{C}_{0}$ as a component. Therefore, by the property of the elliptic sequence (Theorem 3.7 of [4], Proposition 2.1 of [5] and 
Corollary 2.3 of [6]), we have:

(1) $Z_{B_{1+1}}=\tilde{C}_{0}$;

(2) $Z_{B_{0}}^{2} \leqslant \cdots \leqslant Z_{B_{l+1}}^{2}<0$;

(3) $\sum_{i=0}^{l+1} Z_{B_{t}}^{2}=K_{\tilde{X}}^{2}=-n(n$ being the number of blow-ups in $\mu: \tilde{X} \rightarrow \bar{X})$;

(4) $2 \geqslant-\sum_{i=0}^{\prime} Z_{B_{i}}^{2}$.

(Although Yau's proof is for the case when $k=\mathbf{C}$, it is easy to check that (1)-(4) remain true in our situation for any algebraically closed field $k$.) From these we see that all possibilities are as follow:

\begin{tabular}{|l|l|l|l|l|l|}
\hline & $l$ & $Z_{B_{0}}^{2}$ & $Z_{B_{1}}^{2}$ & $\tilde{C}_{0}^{2}$ & $n$ \\
\hline (a) & 1 & -1 & -1 & -1 & 3 \\
\hline (b) & 0 & -2 & & -1 & 3 \\
\hline (c) & 0 & -2 & & -2 & 4 \\
\hline (d) & 0 & -1 & & -1 & 2 \\
\hline
\end{tabular}

Now we can choose a relatively minimal model $\bar{X}$ of $\tilde{X}$ so that $C_{0}^{2}=\tilde{C}_{0}^{2}$. Indeed, if $C_{0}^{2}>\tilde{C}_{0}^{2}$ for an $\bar{X}$, there exists a point $P_{0}$ on $C_{0}$ such that $\mu: \tilde{X} \rightarrow \bar{X}$ factors through the blow-up $\mu_{1}^{\prime}: X_{1}^{\prime} \rightarrow \bar{X}$ with center at $P_{0}$. Hence, there is a morphism $\mu^{\prime}: \tilde{X} \rightarrow \bar{X}^{\prime}$, where $\bar{X}^{\prime}$ is the image of the elementary transformation of $\bar{X}$ with center at $P_{0}$, so $\bar{X}^{\prime}$ is another relatively minimal model of $\tilde{X}$ whose minimal section $C_{0}^{\prime}$ satisfies $C_{0}^{\prime 2}=C_{0}^{2}-1$ :

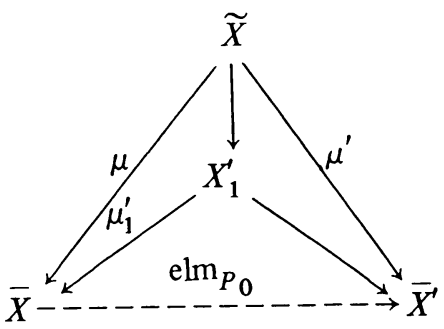

With the assumption of $C_{0}^{2}=\tilde{C}_{0}^{2}$, and using Lemma 2, we can list the possibilities of $\bar{D}, \mu$ and $\tilde{D}$ corresponding to each case in the table above:

(a) and (b). $\bar{D}=2 C_{0}+f_{0}, f_{0}$ is a fibre of $\tilde{\omega}: \bar{X} \rightarrow C$, and the centers of the blow-ups $\mu$ lie on $\operatorname{supp}\left(f_{0}\right) \backslash \operatorname{supp}\left(C_{0}\right)$. Hence $\tilde{D}=2 \tilde{C}_{0}+\tilde{f}_{0}$. ( always denotes the proper transform on $\tilde{X}$.)

(c-1)-(c-3). $\bar{D}=2 C_{0}+f_{1}+f_{2}, f_{1}$ and $f_{2}$ are disjoint fibres of $\tilde{\omega}$.

(c-4) and (c-5). $\bar{D}=2 C_{0}+2 f_{1}, f_{1}$ is a fibre of $\tilde{\omega}$.

In (c-1)-(c-5), $\mu$ and $\tilde{D}$ are described in the following figures: 
(c-1)

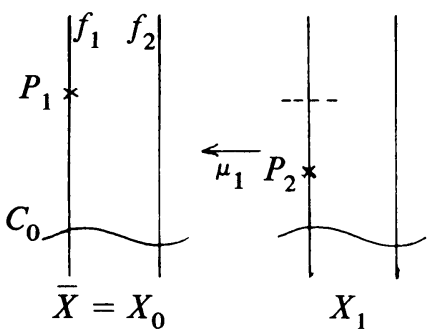

(c-2)
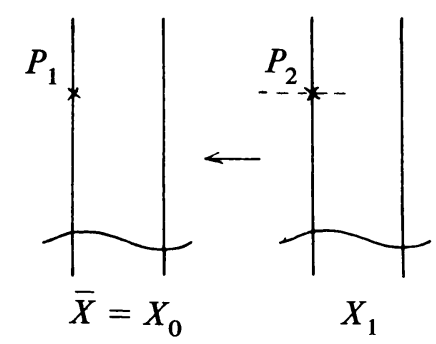

(c-3)
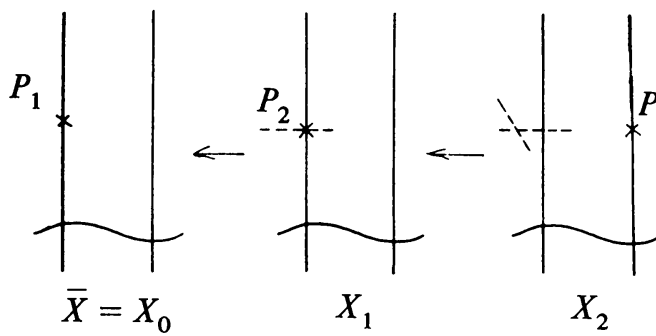

$X_{2}$

$X_{2}$

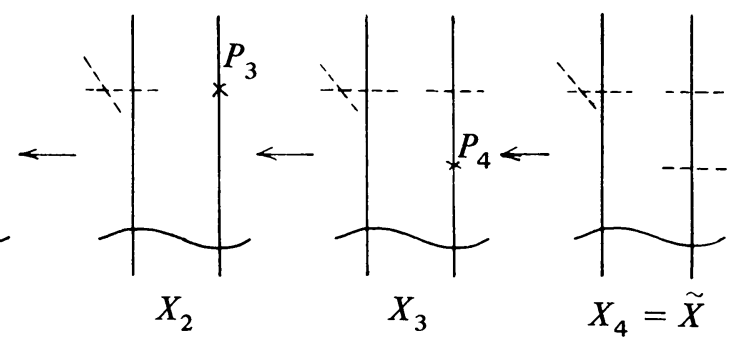

(c-4)

$C_{0}$

$f_{1}$
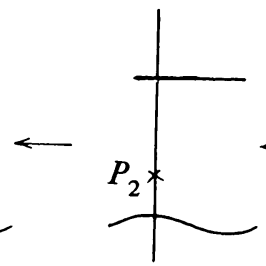

$\bar{X}=X_{0}$

$X_{1}$

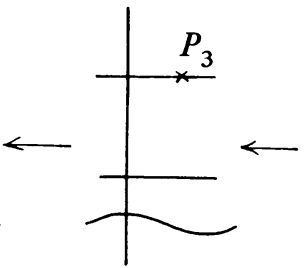

$X_{2}$

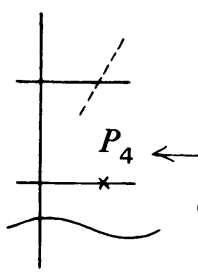

$x_{3}$

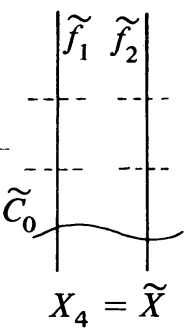

$X_{3}$

$X_{4}=\widetilde{X}$

$X_{4}=\tilde{X}$

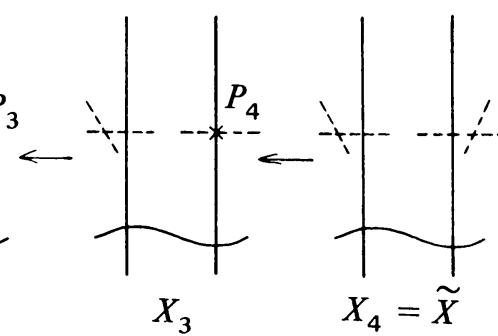

$f_{1}$
$P_{1}$

(c-5)

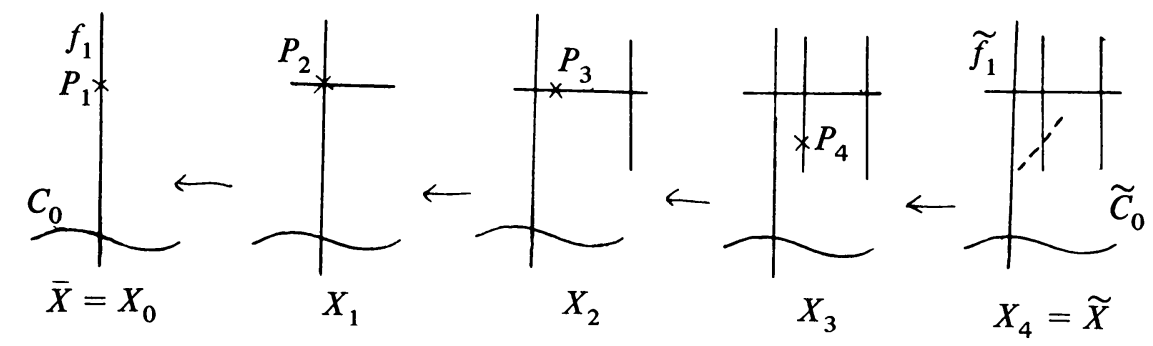


where $P_{i}$ is the center of $\mu_{i}$ (the order of the blow-ups may be changed), the bold lines denote the support of the effective anticanonical divisor $D_{i}$ on each step, while the dotted lines are the exceptional curves of the blow-ups which do not appear in $D_{i}$.

(d). $\bar{D}=2 C_{0}+f_{0}, f_{0}$ is a fibre of $\tilde{\omega}$, the centers of $\mu$ lie on $\operatorname{supp}\left(f_{0}\right) \backslash \operatorname{supp}\left(C_{0}\right)$, and hence $\tilde{D}=2 \tilde{C}_{0}+\tilde{f}_{0}$.

It remains to show that cases (a) and (b) do not occur. For (a), since $l=1, \bar{D}$ must have at least three irreducible components by a property of the elliptic sequence: $\operatorname{supp}\left(\tilde{C}_{0}\right)=\operatorname{supp}\left(Z_{B_{2}}\right) \subset \operatorname{supp}\left(Z_{B_{1}}\right) \subset \operatorname{supp}\left(Z_{B_{0}}\right)=\operatorname{supp}(\tilde{D})$, a contradiction. For (b), let $r_{i}$ be the multiplicity of $H_{i}$ at $P_{i}(1 \leqslant i \leqslant 3)$ and $\bar{H}=m C_{0}+m f(f$ denotes a fibre and $m \in \mathbf{Z}$ ). Then we have

$$
4=\tilde{H}^{2}=\bar{H}^{2}-\sum r_{i}^{2}=m^{2}-\sum r_{i}^{2}
$$

and

$$
0=\tilde{H} \cdot \tilde{f}_{0}=\bar{H} \cdot f_{0}-\sum r_{i}=m-\sum r_{i}
$$

Hence

$$
4=\left(\sum r_{i}\right)^{2}-\sum r_{i}^{2}=2\left(r_{1} r_{2}+r_{2} r_{3}+r_{3} r_{1}\right) .
$$

Since $r_{i} \geqslant 1(1 \leqslant i \leqslant 3)$, we get a contradiction. Q.E.D.

2. Main results. In this section, we assume the ground field $k$ is algebraically closed and has characteristic 0 .

Let $Y_{n} \stackrel{\mu_{n}}{\rightarrow} Y_{n-1} \stackrel{\mu_{n-1}}{\rightarrow} \cdots \stackrel{\mu_{1}}{\rightarrow} Y_{0}=Y$ be a sequence of blow-ups where $Y$ is a nonsingular surface. Let $P_{i} \in Y_{i-1}$ denote the center of $\mu_{i}$, and $E_{i} \subset Y_{i}$ the exceptional curve for $\mu_{i}(1 \leqslant i \leqslant n)$. Then we call $\left(P_{1}, \ldots, P_{n}\right)$ a sequence of points on $Y$ admitting infinitely near points, and the above sequence of blow-ups is called the blow-up of $\left(P_{1}, \ldots, P_{n}\right)$. For a divisor $D$ on $Y$ and nonnegative integers $m_{i}(1 \leqslant i \leqslant n)$, we denote by $\left|D-m_{1} P_{1}-\cdots-m_{n} P_{n}\right|$ the linear subsystem of $|D|$ consisting of elements $D^{\prime} \in|D|$ such that

$$
\mu_{n}^{*}\left(\mu_{n-1}^{*}\left(\cdots\left(\mu_{1}^{*}\left(D^{\prime}\right)-m_{1} E_{1}\right) \cdots\right)-m_{n-1} E_{n-1}\right)-m_{n} E_{n}
$$

remain effective on $Y_{n}$. When there is no danger of confusion, we also denote by $P_{i}$ the image on $Y$ of the point $P_{i} \in Y_{i-1}$.

Let $C$ be a nonsingular curve and $E$ a vector bundle of rank 2 on $C$. Assume $E$ is the direct sum of two line bundles. Then the ruled surface $\mathbf{P}(E) \rightarrow C$ has a section which is disjoint from a minimal section. We denote such a section by $C_{1}$; we denote a minimal section by $C_{0}$, as before. In general these sections are not uniquely determined, but we fix a pair $\left(C_{0}, C_{1}\right)$ once and for all on $\mathbf{P}(E)$ unless otherwise mentioned.

THEOREM 1. Let $C$ be a nonsingular elliptic curve and let $L$ be an invertible sheaf of degree 2 on $C$. Let $\bar{X}=\mathbf{P}\left(O_{C} \oplus L\right) \stackrel{\dot{\omega}}{\rightarrow} C$ be the induced ruled surface. Fix an effective divisor $\bar{D} \in\left|-K_{\bar{X}}\right|$ and take a sequence of points $\left(P_{1}, P_{2}, P_{3}\right)$ on $\bar{X}$ admitting infinitely 
near points. Assume the following property for $\left(P_{1}, P_{2}, P_{3}\right)$ :

If $\bar{D}=C_{0}+C_{1}^{\prime}$, where $C_{1}^{\prime}$ is a section of $\tilde{\omega}$, then each $P_{i}$ lies on $C_{1}^{\prime}$; and if $\bar{D}=2 C_{0}+f_{1}+f_{2}$, where the $f_{i}$ 's are fibres, then the position of $P_{1}, P_{2}, P_{3}$ is one of the types (c-1)-(c-5). In each case,

$(*)$ there is no section $C^{\prime}$ of $\tilde{\omega}$ such that $C^{\prime} \sim C_{1}, C^{\prime} \$ \bar{D}$ and $P_{i}$, $P_{j} \in C^{\prime}$ for some $i, j(1 \leqslant i<j \leqslant 3)$.

Then

(1) there exists a unique point $P_{4}$ on $\bar{X}$ (possibly an infinitely near point) such that $\left|2 C_{1}-P_{1}-P_{2}-P_{3}\right|=\left|2 C_{1}-P_{1}-P_{2}-P_{3}-P_{4}\right|$.

Let $\mu: \tilde{X} \rightarrow \bar{X}$ be the blow-up of $\left(P_{1}, P_{2}, P_{3}, P_{4}\right)$ and $\tilde{H}$ the proper transform by $\mu$ of a general member in $\left|2 C_{1}-P_{1}-P_{2}-P_{3}-P_{4}\right|$. Then we have

(2) $\mathrm{Bs}|\tilde{H}|=\varnothing$;

(3) $\operatorname{dim}|\tilde{H}|=3, \tilde{H}^{2}=4$;

(4) $\tilde{H}$ is a nonhyperelliptic curve.

Therefore, $\tilde{H}$ defines a birational morphism from $\tilde{X}$ to a normal quartic surface $X \subset \mathbf{P}^{3}$ with singularities of type $2 \tilde{E}_{7}, E 1+A_{1}+A_{1}, E 1+D(3)$ or $E 1+D(4)$.

Conversely, any quartic surface of elliptic ruled type with at least one of these four types of singularities is obtained by the construction above.

REMARK. Without condition $(*),(1)-(3)$ of Theorem 1 hold. But in this case $\tilde{H}$ turns out to be a hyperelliptic curve so $\tilde{H}$ defines a morphism of degree 2 onto a normal quadric surface in $\mathbf{P}^{3}$ (cf. Lemmas 6 and 7 in $\S 3$ ). Furthermore, it will be shown in $\S 5$ that the case (c-3) occurs only if we remove condition $(*)$.

THEOREM 2. Let $C$ be a nonsingular elliptic curve and $L$ an invertible sheaf of degree 1 on $C$. Let $\bar{X}=\mathbf{P}\left(O_{C} \oplus L\right) \stackrel{\bar{\omega}}{\rightarrow} C$ be the induced ruled surface. Fix an effective divisor $\bar{D} \in\left|-K_{\bar{X}}\right|$ and take a point $P_{1}$ on $\bar{X}$ such that:

If $\bar{D}=C_{0}+C_{1}^{\prime}$, where $C_{1}^{\prime}$ is a section of $\tilde{\omega}$, then $P_{1} \in \operatorname{supp}\left(C_{1}^{\prime}\right)$, $O_{C_{1}^{\prime}}\left(P_{1}\right) \nsucceq L$, and

$$
O_{C_{1}^{\prime}}\left(2 P_{1}\right) \not L^{2}
$$

(identifying $C_{1}^{\prime}$ with $C$ via $\tilde{\omega}$ ); and if $\bar{D}=2 C_{0}+f_{0}$, where $f_{0}$ is a fibre of $\tilde{\omega}$, then $P_{1} \in \operatorname{supp}\left(f_{0}\right) \backslash \operatorname{supp}\left(C_{0}+C_{1}\right)$.

Then

(1) there exists a unique point $P_{2}$ on $\bar{X}$ (possibly an infinitely near point) such that $\left|3 C_{1}-2 P_{1}\right|=\left|3 C_{1}-2 P_{1}-P_{2}\right|$.

Let $\mu: \tilde{X} \rightarrow \bar{X}$ be the blow-up of $\left(P_{1}, P_{2}\right)$ and $\tilde{H}$ the proper transform by $\mu$ of $a$ general member in $\left|3 C_{1}-2 P_{1}-P_{2}\right|$. Then we have:

(2) $\operatorname{Bs}|\tilde{H}|=\varnothing$;

(3) $\operatorname{dim}|\tilde{H}|=3, \tilde{H}^{2}=4$;

(4) $\tilde{H}$ is a nonhyperelliptic curve.

Therefore, $\tilde{H}$ defines a birational morphism from $\tilde{X}$ to a normal quartic surface $X \subset \mathbf{P}^{3}$ with singularities of type $2 \tilde{E}_{8}$ or $E 1+A_{1}$. 
Conversely, any quartic surface of elliptic ruled type with at least one of these two types of singularities is obtained by the construction above.

REMARK. Even if we take $\bar{D}=C_{0}+C_{1}^{\prime}$ and $P_{1} \in \operatorname{supp}\left(C_{1}^{\prime}\right)$ such that $O_{C_{1}^{\prime}}\left(P_{1}\right) \not L$ and $O_{C_{1}^{\prime}}\left(2 P_{1}\right) \cong L^{2},(1)-(3)$ of Theorem 2 hold. But then $\tilde{H}$ is hyperelliptic and the image of the morphism defined by $\tilde{H}$ is a nonsingular quadric surface in $\mathbf{P}^{3}$ (cf. Lemma 9 and the end of $\S 4$ ).

COROLlary. Let $X$ be a quartic surface of elliptic ruled type. Then the singular set of $X$ is one of the following types, and each of them really occurs:

$$
\begin{aligned}
& \left\{2 \tilde{E}_{7}, \text { a subgraph of } A_{3}\right\}, \quad\left\{E 1+A_{1}+A_{1}, \text { a subgraph of } A_{1}\right\}, \\
& \{E 1+D(3)\}, \quad\{E 1+D(4)\}, \quad\left\{2 \tilde{E}_{8}\right\}, \quad\left\{E 1+A_{1}\right\} .
\end{aligned}
$$

3. Proof of Theorem 1. $\bar{X}, \bar{D}, P_{1}, P_{2}$ and $P_{3}$ are as in the theorem, but at first we do not assume condition $(*)$.

Proofs of (1)-(3). Set $\Lambda=\left|2 C_{1}-P_{1}-P_{2}-P_{3}\right|$. Since $\Lambda \varsubsetneqq\left|2 C_{1}-P_{1}-P_{2}\right|$ $\subsetneq\left|2 C_{1}-P_{1}\right| \subsetneq\left|2 C_{1}\right|$, as is easily shown in each case, and since $\operatorname{dim}\left|2 C_{1}\right|=$ $\operatorname{dim} H^{0}\left(C, O_{C} \oplus L \oplus L^{2}\right)-1=6$, we have $\operatorname{dim} \Lambda=3$. First we take $\bar{D}=C_{0}+C_{1}^{\prime}$, where $C_{1}^{\prime}$ is a section. By $C_{1}^{\prime} \sim C_{1}$, we may assume $\bar{D}=C_{0}+C_{1}$. Since $2 C_{1} \cdot C_{1}=4$ and $C_{1}$ is an elliptic curve, we can define a unique point $P_{4}$ lying on the proper transform of $C_{1}$ on the blow-up of $\left(P_{1}, P_{2}, P_{3}\right)$ of $\bar{X}$ such that

$$
\Lambda=\left|2 C_{1}-P_{1}-P_{2}-P_{3}-P_{4}\right| \text {. }
$$

Let $f_{i}$ denote the fibre on $\bar{X}$ through $P_{i}(1 \leqslant i \leqslant 4)$. Since

$$
O_{C_{1}}\left(P_{1}+P_{2}+P_{3}+P_{4}\right) \cong O_{C_{1}}\left(2 C_{1}\right) \cong L^{2},
$$

identifying $C_{1}$ and $C$ via $\tilde{\omega}$, we find the following four elements of $\Lambda: 2 C_{0}+\sum_{i=1}^{4} f_{i}$, $C_{0}+f_{5}+f_{6}+C_{1}, C_{0}+f_{7}+f_{8}+C_{1}$ and $2 C_{1}$, where the $f_{j}$ 's $(5 \leqslant j \leqslant 8)$ are fibres disjoint from each other and from $f_{i}(1 \leqslant i \leqslant 4)$ such that $f_{5}+f_{6}, f_{7}+f_{8} \in\left|\tilde{\omega}^{*} L\right|$. Let $s_{0}, \ldots, s_{3} \in H^{0}\left(\bar{X}, O_{\bar{X}}\left(2 C_{1}\right)\right)$ denote the defining equations of the above four divisors. Obviously the $s_{i}$ 's are linearly independent, and hence form a basis of $\Lambda$. Therefore, letting $\bar{H}$ be a general member of $\Lambda$ we define $\mu: \tilde{X} \rightarrow \bar{X}$ and $\tilde{H}$ as in the theorem and deduce that:

the base points of $\Lambda$ are exactly $P_{1}, \ldots, P_{4}$;

Bs $|\tilde{H}|=\varnothing$;

$\operatorname{dim}|\tilde{H}|=\operatorname{dim} \Lambda=3$ and $\bar{H}$ is nonsingular at $P_{1}, \ldots, P_{4}$.

Thus (1)-(3) follow. Next suppose $\bar{D}=2 C_{0}+f_{1}+f_{2}$, where $f_{1}$ and $f_{2}$ are fibres. $\left(f_{2}\right.$ may coincide with $f_{1}$.) Let $\bar{H}$ be a general member of $\Lambda$ so $2 C_{0}+2 f_{1}+2 f_{2}$, $2 C_{0}+f_{1}+f_{2}+f_{3}+f_{4}, C_{0}+f_{1}+f_{2}+C_{1}^{\prime}$ and $\bar{H}$ correspond to a basis of $\Lambda$, where $f_{3}$ and $f_{4}$ are fibres disjoint from $f_{1}$ and $f_{2}$, with $f_{3}+f_{4} \in\left|\tilde{\omega}^{*} L\right|$, and $C_{1}^{\prime}$ is a section disjoint from $P_{i}(1 \leqslant i \leqslant 4)$ and $C_{0}$. In particular, $\bar{H}$ has no component in common with $\bar{D}$. Therefore we can define $P_{4}$ uniquely as the intersection of the proper transform of $\bar{H}$ in $X_{3}$ with $D_{3}$ in the notation of the figures (c-1)-(c-5) in $\S 1$. Let $\mu$ : $\tilde{X} \rightarrow \bar{X}$ and $\tilde{H}$ be as in the theorem. Then we can show that $|\tilde{H}|$ has no basepoint. For example, in the case of (c-1), $\bar{H}$ and $C_{0}+f_{1}+f_{2}+f_{3}+f_{4}$ must have distinct 
tangents at $P_{i}$ so $|\tilde{H}|$ has no basepoint over $P_{i}$. After this, we trace the proof of the above case and obtain (1)-(3).

Let $X$ denote the image of $\tilde{X}$ under the morphism $\Phi_{|\tilde{H}|}$ associated with $\tilde{H}$. Then $X$ is a hypersurface in $\mathbf{P}^{3}$ of degree 4 or 2 by (3), and $X$ is normal because the pull-back of a general hyperplane section of $X$ is a nonsingular curve $\tilde{H}$. It is clear by our construction that there is a unique effective anticanonical divisor $\tilde{D}$ on $\tilde{X}$, and the configuration of $\tilde{D}$ is $2 \tilde{E}_{7}, E 1+A_{1}+A_{1}, E 1+D(3)$ or $E 1+D(4)$ according to our choice of $\bar{D}, P_{1}, P_{2}$ and $P_{3}$. Moreover, by a direct computation, we see that $\operatorname{supp}(\tilde{D})$ consists of one or two of the connected components of the exceptional set for $\Phi_{|\tilde{H}|}$. By $\tilde{H} \cdot K_{\tilde{X}}=0$, the restriction of $\Phi_{\mid \tilde{H}}$ to $\tilde{H}$ is the canonical map of $\tilde{H}$; therefore, $X$ is a quartic surface if and only if $\tilde{H}$ is nonhyperelliptic. We will show a criterion for this property in a generalized situation.

Let $\Sigma_{0}$ and $\Sigma_{2}^{0}$ denote a nonsingular quadric surface in $\mathbf{P}^{3}$ and a cone over a nonsingular conic in $\mathbf{P}^{3}$, respectively, and let $v$ denote the vertex of $\Sigma_{2}^{0}$. Let $Y$ be a nonsingular surface birationally equivalent to an elliptic ruled surface. Suppose there is an effective anticanonical divisor $D$ of $Y$ such that every irreducible component of $D$ has self-intersection number greater than -4 . Moreover, suppose there exists on $Y$ a nonsingular curve $H$ of genus 3 such that $\mathrm{Bs}|H|=\varnothing, \operatorname{dim}|H|=3$ and $H \cdot D=0$, so we have a morphism $\Phi=\Phi_{|H|}$ from $Y$ onto a normal quartic surface in $\mathbf{P}^{3}$ or a $\Sigma_{0}$ or a $\Sigma_{2}^{0}$. Since $H \cdot D=0, \Phi(D)$ consists of finite points.

Lemma 5. Assume $\Phi(Y)=\Sigma_{2}^{0}$. Then $v \notin \Phi(D)$.

Proof. Let $\pi_{1}: \Sigma_{2}=\mathbf{P}\left(O_{\mathbf{P}^{\prime}} \oplus O_{\mathbf{P}^{\prime}}(-2)\right) \rightarrow \Sigma_{2}^{0}$ be the blow-up with center at $v$, and let $\Gamma_{0}$ be the exceptional rational curve for $\pi_{1}$. Let $\Gamma$ be a general hyperplane section of $\Sigma_{2}^{0}$ and let $\Gamma_{1}$ denote the proper transform of $\Gamma$ on $\Sigma_{2}$. We may assume that $\Phi(H)=\Gamma$. Then we get the following commutative diagram:

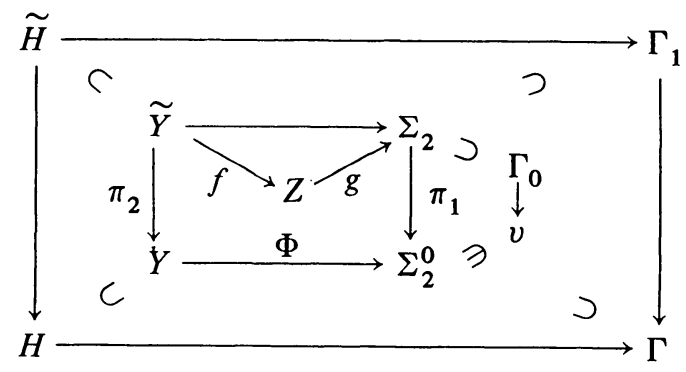

where $\pi_{2}: \tilde{Y} \rightarrow Y$ is a minimal sequence of blow-ups such that the induced map $\tilde{Y} \rightarrow \Sigma_{2}$ becomes a morphism, $Z$ is the Stein factorization of $\tilde{Y} \rightarrow \Sigma_{2}$, and $\tilde{H}$ is the proper transform of $H$. Let $B$ denote the branch locus of $g$. Then $B \sim 2\left(n \Gamma_{0}+m f\right)$ for some $n, m \in \mathbf{Z}$ where $f$ denotes a fibre of the ruling on $\Sigma_{2}$. Since $\tilde{H} \rightarrow \Gamma_{1}$ is a morphism of degree 2 between nonsingular curves of genus 3 and 0 , respectively, we have $B \cdot \Gamma_{1}=8$ and hence $B \sim 2 n \Gamma_{0}+8 f$. Furthermore, since $B$ is reduced,

$$
-2=\Gamma_{0}^{2} \leqslant \Gamma_{0} \cdot B=-4 n+8 .
$$

If $n=0$, then $Z$ is a ruled surface of genus 3, and hence a contradiction. Therefore we have $n=1$ or 2 . 
Assume $\Phi(D) \ni v$. Then there exists a nonsingular elliptic curve $C \leqslant D$ such that $\Phi(C)=v$ by Lemmas 2 and 3. Let $\tilde{C}$ denote the proper transform of $C$ on $\tilde{Y}$. If $f(\tilde{C})$ is a curve, then clearly $\tilde{Y}=Y$, and $g^{-1}\left(\Gamma_{0}\right)=f(\tilde{C}) \cong \tilde{C}=C$ (consider the configuration of $D$ ). Hence, we get a contradiction because $C^{2}=2 \cdot \Gamma_{0}^{2}=-4$. Thus $f(\tilde{C})$ is a point, and so $Z$ has a singular point on $g^{-1}\left(\Gamma_{0}\right)$ which is not a rational double point.

Suppose $n=1$. Then $B \cdot f=2$. From this we easily see that $Z$ has at worst rational double points. Suppose $n=2$; then $B \sim 4 \Gamma_{0}+8 f$. If $\Gamma_{0} \$ B$, then $Z$ is smooth over $\Gamma_{0}$ since $\Gamma_{0} \cdot B=0$. Hence $B=\Gamma_{0}+B_{0}$ for some reduced effective divisor $B_{0}$ not containing $\Gamma_{0}$. But since $B_{0} \cdot \Gamma_{0}=2$, in this case also $Z$ has only rational double points over $\Gamma_{0}$, a contradiction. Q.E.D.

LEMMA 6. $\Phi(Y)$ is a normal quartic surface if and only if there is no curve $C$ on $Y$ satisfying

(**) $\quad C$ is nonsingular, elliptic, $C \cdot H=2$ and $\operatorname{supp}(C) \cap \operatorname{supp}(D)=\varnothing$.

Proof. Suppose $\Phi(Y)$ is a quartic surface and there is a curve $C$ on $Y$ satisfying $(* *)$. Then $\Phi(C)$ is a curve and is birationally equivalent to $C$, because $C \cdot H>0$ and $\Phi$ is a birational morphism. But by $\Phi(C) \cdot h=C \cdot H=2$ for a hyperplane section $h$ of $\Phi(Y), \Phi(C)$ must be a rational curve, and hence a contradiction. Next suppose $\Phi(Y)=\Sigma_{0}$. Let us take a general fibre $l$ of one of the two rulings on $\Sigma_{0}$. Then it is easy to verify that the proper transform of $l$ on $Y$ satisfies $(* *)$. Finally suppose $\Phi(Y)=\Sigma_{2}^{0}$ and let $l$ be a general generating line of $\Sigma_{2}^{0}$. Then, by Lemma 5 , $l$ and $\Phi(D)$ are disjoint. It follows that the proper transform of $l$ on $Y$ satisfies $(* *)$. Q.E.D.

Now we return to the proof of Theorem 1. To prove that $X$ is really a quartic surface under the assumption of $(*)$, it suffices to apply Lemma 6 to the case of $Y=\tilde{X}$ (and so $H=\tilde{H}, D=\tilde{D}$ ) and combine it with the following

LEMma 7. The nonexistence of curves on $\tilde{X}$ satisfying $(* *)$ is equivalent to condition $(*)$.

Proof. If there is a section $C^{\prime}$ on $\bar{X}$ such that $C^{\prime} \sim C_{1}, C^{\prime} \$ \bar{D}$ and $C^{\prime} \ni P_{i}, P_{j}$ for some $i, j(1 \leqslant i<j \leqslant 3)$, then clearly the proper transform of $C^{\prime}$ on $\tilde{X}$ satisfies $(* *)$.

Conversely, suppose there exists on $\tilde{X}$ a curve $\tilde{C}$ satisfying (**). Put $C^{\prime}=\mu(\tilde{C})$, and $r_{i}=$ mult $_{P_{1}} C^{\prime}(1 \leqslant i \leqslant 4)$. Since $\tilde{C} \cdot \tilde{C}_{0}=0$, we have $C^{\prime} \cdot C_{0}=0$, so $C^{\prime}=m C_{1}$ for some $m \in \mathbf{Z}$. Therefore we have

$$
\begin{aligned}
& 2=\tilde{C} \cdot \tilde{H}=C^{\prime} \cdot \bar{H}-\sum r_{i}=4 m-\sum r_{i}, \\
& 0=\tilde{C} \cdot \tilde{D}=C^{\prime} \cdot \bar{D}-\sum r_{i}=2 m-\sum r_{i} .
\end{aligned}
$$

So we get $m=1$ and $\sum r_{i}=2$. We may assume $r_{1}=1$ and $r_{2}=0$. If $r_{3}=1$ and $r_{4}=0$, there is nothing to prove. Assume $r_{3}=0$ and $r_{4}=1$. This is the case of $\bar{D}=C_{0}+C_{1}^{\prime}$ or $(\mathrm{c}-1)$ or $(\mathrm{c}-2)$. Let $C^{\prime \prime}$ be a section which is disjoint from $C_{0}$ and passes through $P_{2}$, and let $\left.\bar{D}\right|_{C^{\prime \prime}}=P_{2}+P_{3}^{\prime}$. Since $P_{3} \neq P_{4}$ as points on $\bar{X}$, we can define $\Lambda^{\prime}=\left|2 C_{1}-P_{1}-P_{2}-P_{4}\right|$ and we have $C^{\prime}+C^{\prime \prime} \in \Lambda^{\prime}$. But, in fact, $\Lambda^{\prime}$ 
coincides with $\Lambda=\left|2 C_{1}-P_{1}-P_{2}-P_{3}-P_{4}\right|$. Therefore, $P_{3}^{\prime}=P_{3}$ and hence $C^{\prime \prime}$ is a desired section. Q.E.D.

Finally, we prove the second part of the theorem. Let $X$ be a quartic surface of elliptic ruled type with at least $2 \tilde{E}_{7}, E 1+A_{1}+A_{1}, E 1+D(3)$ or $E 1+D(4)$ as singularities. Let $\pi: \tilde{X} \rightarrow X$ be the minimal resolution of $X$. Then from the proof of Proposition 2, we see there is a ruled surface $\bar{X}=\mathbf{P}(E) \stackrel{\tilde{\omega}}{\rightarrow} C$ over an elliptic curve $C$ such that $C_{0}^{2}=-2$, in particular, $E$ splits (the notation is the same as before), and there exists a sequence of points $\left(P_{1}, P_{2}, P_{3}, P_{4}\right)$ on $\bar{X}$ such that $\tilde{X}$ is the blowing-up of $\bar{X}$ at the $P_{i}$ 's. This blowing-up $\tilde{X} \rightarrow \bar{X}$ is the sequence in Lemma 2. We have already shown that $\left(P_{1}, P_{2}, P_{3}\right)$ must satisfy the conditions described in the theorem, and hence, we only have to show the following

Lemma 8. Let $\bar{H} \subset \bar{X}$ be as in Lemma 2. Then $\bar{H} \in\left|2 C_{1}\right|$.

Proof. In the notation of Lemma $2, \bar{H} \equiv m C_{1}(m \in \mathbf{Z})$. Indeed, since $\tilde{H} \cdot \tilde{C}_{0}=0$, $\bar{H}$ is numerically equivalent to some multiple $m C_{1}$. We show that $m=2$.

Case 1. $X$ has a singularity of $p_{g}=2$. We may assume the order of $P_{1}, P_{2}, P_{3}, P_{4}$ is the same as one of the figures (c-1)-(c-5) in $\S 1$, and we use the notation there. Put $r_{i}=$ mult $_{P_{i}} H_{i}$ for $1 \leqslant i \leqslant 4$. Then we have

$$
4=\tilde{H}^{2}=\bar{H}^{2}-\sum r_{i}^{2}=2 m^{2}-\sum r_{i}^{2} .
$$

On the other hand, since the proper transform of $\bar{H}$ and $f_{1}$ must be disjoint after blowing-up $\left(P_{1}, P_{2}\right)$, we get

$$
r_{1}+r_{2}=\bar{H} \cdot f_{1}=m .
$$

Replacing $\left\{P_{1}, P_{2}, f_{1}\right\}$ with $\left\{P_{3}, P_{4}, f_{2}\right\}$, if necessary, in the case of (c-1)-(c-3), we can assume $r_{1}^{2}+r_{2}^{2} \geqslant r_{3}^{2}+r_{4}^{2}$. Therefore, we have

$$
4=2\left(r_{1}+r_{2}\right)^{2}-\sum r_{i}^{2}=\left(r_{1}^{2}+r_{2}^{2}-r_{3}^{2}-r_{4}^{2}\right)+4 r_{1} r_{2} \geqslant 4 r_{1} r_{2} .
$$

It follows that $r_{1}=r_{2}=1$, so $m=2$.

Case 2. $X$ has $2 \tilde{E}_{7}$. Let $r_{i}=$ mult $_{P_{i}} H_{i}(1 \leqslant i \leqslant 4)$. Then we have

$$
4=\tilde{H}^{2}=\bar{H}^{2}-\sum r_{i}^{2}=2 m^{2}-\sum r_{i}^{2}, \quad \sum r_{i}=\bar{H} \cdot C_{1}=2 m .
$$

Therefore we get $m \neq 3$, because the above two equations have no positive integral solution $\left(r_{1}, r_{2}, r_{3}, r_{4}\right)$ for $m=3$. Next, since $P_{1}, \ldots, P_{4}$ lie on $\operatorname{supp}\left(C_{1}\right)$, we can find another relatively minimal model $\tilde{X} \stackrel{\mu^{\prime}}{\rightarrow} \bar{X}^{\prime}$ such that $\bar{X}^{\prime}=\mathbf{P}\left(O_{C} \oplus L^{\prime}\right)$, where $L^{\prime}$ is an invertible sheaf of degree 0 on $C$. Then there are two disjoint sections $C_{0}^{\prime}, C_{1}^{\prime}$ of $\tilde{\omega}^{\prime}$ : $\bar{X}^{\prime} \rightarrow C$ and four points $P_{1}^{\prime}, P_{2}^{\prime} \in C_{0}^{\prime}, P_{3}^{\prime}, P_{4}^{\prime} \in C_{1}^{\prime}$ admitting infinitely near points such that $\mu^{\prime}$ is the blow-up of $\left(P_{1}^{\prime}, P_{2}^{\prime}, P_{3}^{\prime}, P_{4}^{\prime}\right)$ and $\mu^{\prime}\left(\tilde{C}_{0}+\tilde{C}_{1}\right)=C_{0}^{\prime}+C_{1}^{\prime}$. We may assume $\left.O_{\bar{X}^{\prime}}\left(C_{1}^{\prime}\right)\right|_{C_{1}^{\prime}} \cong L^{\prime}$ and $\left.O_{\bar{X}^{\prime}}\left(C_{0}^{\prime}\right)\right|_{C_{0}^{\prime}} \cong L^{\prime-1}$. Set $\overline{H^{\prime}}=\mu^{\prime}(\tilde{H})$ and $r_{i}^{\prime}=$ mult ${\overline{P^{\prime}}}_{\bar{H}^{\prime}}$. Then $\bar{H}^{\prime} \equiv m C_{0}^{\prime}+n f$ for some $n \in \mathbf{Z}$ where $f$ is a fibre of $\tilde{\omega}^{\prime}$. By $\tilde{H} \cdot \tilde{C}_{0}=\tilde{H} \cdot \tilde{C}_{1}=0$, we get

$$
r_{1}^{\prime}+r_{2}^{\prime}=\bar{H}^{\prime} \cdot C_{0}^{\prime}=n, \quad r_{3}^{\prime}+r_{4}^{\prime}=\bar{H}^{\prime} \cdot C_{1}^{\prime}=n .
$$


We may assume $r_{1}^{\prime} \geqslant r_{3}^{\prime} \geqslant r_{4}^{\prime} \geqslant r_{2}^{\prime}$ so $r_{1}^{\prime 2}+r_{2}^{\prime 2} \geqslant r_{3}^{\prime 2}+r_{4}^{\prime 2}$, and equality holds if and only if $r_{1}^{\prime}=r_{3}^{\prime}$. Then we obtain

$$
4=\tilde{H}^{2}=\bar{H}^{\prime 2}-\sum r_{i}^{\prime 2} \geqslant 2 m\left(r_{1}^{\prime}+r_{2}^{\prime}\right)-2\left(r_{1}^{\prime 2}+r_{2}^{\prime 2}\right),
$$

i.e.

$$
0 \geqslant r_{1}^{\prime}\left(m-r_{1}^{\prime}\right)+r_{2}^{\prime}\left(m-r_{2}^{\prime}\right)-2,
$$

and equality holds if and only if $r_{1}^{\prime}=r_{3}^{\prime}$. Since $m \geqslant \bar{H}^{\prime} \cdot f \geqslant r_{1}^{\prime} \geqslant r_{2}^{\prime} \geqslant 1$, the right-hand side of $(* * *)$ is either (i) -2 , (ii) -1 or (iii) 0 . In the case of (i), we have $m-r_{1}^{\prime}=m-r_{2}^{\prime}=0$ so $r_{1}^{\prime}=r_{3}^{\prime}$, which implies a contradiction. In case (ii), we get $m-r_{1}^{\prime}=0, r_{2}^{\prime}=m-r_{2}^{\prime}=1$ and hence $m=2$. (But it follows that $r_{1}^{\prime}=r_{3}^{\prime}$ because $r_{1}^{\prime}=2$ and $r_{2}^{\prime}=1$, hence this case is impossible.) In (iii), there are three possibilities: (a) $r_{1}^{\prime}=m-r_{1}^{\prime}=r_{2}^{\prime}=m-r_{2}^{\prime}=1$; (b) $m-r_{1}^{\prime}=0, r_{2}^{\prime}=1, m-r_{2}^{\prime}=2$; and (c) $m-r_{1}^{\prime}=0, r_{2}^{\prime}=2, m-r_{2}^{\prime}=1$. In (a) we get $m=2$, and so we are done. In (b) and (c), we get $m=3$, but this value has already been excluded.

Therefore, we conclude that $\bar{H} \equiv 2 C_{1}$, and hence, $\bar{H} \sim 2 C_{1}+\tilde{\omega}^{*}(D)$ for some divisor $D$ on $C$ of degree 0 . Since $\bar{X}=\mathbf{P}\left(O_{C} \oplus L\right)$ for some invertible sheaf $L$ of degree 2 on $C$, we have

$$
\begin{aligned}
\operatorname{dim} H^{0}\left(\bar{X}, O_{\bar{X}}(\bar{H})\right) & =\operatorname{dim} H^{0}\left(C,\left(O_{C} \oplus L \oplus L^{2}\right) \otimes O_{C}(D)\right) \\
& = \begin{cases}7 & \text { if } D \sim 0, \\
6 & \text { if } D \nsim 0,\end{cases}
\end{aligned}
$$

and

$$
\operatorname{dim} H^{0}\left(\bar{X}, O_{\bar{X}}\left(\bar{H}-C_{0}\right)\right)=\operatorname{dim} H^{0}\left(C,\left(L \oplus L^{2}\right) \otimes O_{C}(D)\right)=6 .
$$

Since $\bar{H}$ is irreducible, $|\bar{H}|$ has no fixed component, hence we prove that $D \sim 0$, i.e. $\bar{H} \sim 2 C_{1}$. Q.E.D.

4. Proof of Theorem 2. We proceed in several steps which are almost parallel to the proof of Theorem 1 .

First we prove (1)-(3) without assuming condition $(*)$. Let $\Lambda$ denote the linear system $\left|3 C_{1}-2 P_{1}\right|$ on $\bar{X}$. Then we get $\operatorname{dim} \Lambda=3$ because $\operatorname{dim}\left|3 C_{1}\right|=6$, $\operatorname{dim}\left|3 C_{1}-P_{1}\right|=5$, and $\left|3 C_{1}-P_{1}\right|$ contains two elements whose tangents at $P_{1}$ are different from each other. If $\bar{D}=C_{0}+C_{1}^{\prime}$ where $C_{1}^{\prime}$ is a section of $\tilde{\omega}$, we may assume $C_{1}^{\prime}=C_{1}$. Consider the following elements in $\Lambda: 3 C_{1}, 2 C_{1}+C_{0}+f_{0}, C_{1}+$ $2 C_{0}+f_{1}+f_{1}^{\prime}$ and $3 C_{0}+2 f_{1}+f_{2}$, where the $f_{i}^{\prime}$ 's and $f_{1}^{\prime}$ are fibres of $\tilde{\omega}$ such that $f_{0} \in\left|\tilde{\omega}^{*} L\right|, f_{1}$ passes through $P_{1}, f_{1}+f_{1}^{\prime} \in\left|\tilde{\omega}^{*} L^{2}\right|$ and $2 f_{1}+f_{2} \in\left|\tilde{\omega}^{*} L^{3}\right|$. Clearly the defining equations of these four divisors are linearly independent in $H^{0}\left(\bar{X}, O_{\bar{X}}\left(3 C_{1}\right)\right)$ and so form a basis of $\Lambda$. Let $P_{2}$ denote the intersection of $f_{2}$ with $C_{1}$, by the identification of $P_{2}$ with a point on the proper transform of $C_{1}$ in the blow-up of $\bar{X}$ with center at $P_{1}$ if $f_{1}=f_{2}$. Then the basepoints of $\Lambda$ are exactly $2 P_{1}+P_{2}$ in the infinitely near sense and we deduce (1)-(3) as in the proof of Theorem 1. If $\bar{D}=2 C_{0}+f_{0}$, let $\bar{H}$ denote a general member of $\Lambda$ such that $3 C_{0}+3 f_{0}, 2 C_{0}+2 f_{0}+C_{1}, C_{0}+f_{0}+C_{2}$ and $\bar{H}$ correspond to a basis of $\Lambda$, where 
$C_{2}$ is an element of $\left|2 C_{1}\right|$ passing through $P_{1}$ and not containing $C_{0}$. Let $P_{0}$ [resp. $P_{2}, P_{3}, P_{\infty}$ ] denote the point on $\bar{X}$ such that the intersection of $f_{0}$ with $C_{0}$ [resp. $\bar{H}, C_{2}, C_{1}$ ] is $P_{0}$ [resp. $2 P_{1}+P_{2}, P_{1}+P_{3}, P_{\infty}$ ]. Then we will show that $P_{0}, P_{1}, P_{2}, P_{3}$ and $P_{\infty}$ are different from each other. By our hypothesis there is an affine coordinate $t$ on $f_{0} \cong \mathbf{P}^{1}$ such that $t=0,1, \infty$ at $P_{0}, P_{1}, P_{\infty}$, respectively. Let $t=\alpha$ at $P_{2}$ and $t=\beta$ at $P_{3}$ for some $\alpha, \beta \in k \sqcup\{\infty\}$. Set $\lambda=\left|3 C_{1}\right|_{\mid f_{0}}$. From

$$
0 \rightarrow O_{\bar{X}}\left(3 C_{1}-f_{0}\right) \rightarrow O_{\bar{X}}\left(3 C_{1}\right) \rightarrow O_{f_{0}}\left(3 C_{1}\right) \rightarrow 0,
$$

we have $\operatorname{dim} \lambda=2$. Let $f_{i}(1 \leqslant i \leqslant 5)$ be fibres disjoint from $f_{0}$ such that $f_{1}+f_{2} \in$ $\left|\tilde{\omega}^{*} L^{2}\right|$ and $f_{3}+f_{4}+f_{5} \in\left|\tilde{\omega}^{*} L^{3}\right|$. Then $3 C_{1}, C_{1}+2 C_{0}+f_{1}+f_{2}$ and $3 C_{0}+f_{3}+f_{4}$ $+f_{5}$ cut on $f_{0}$ three elements in $\lambda: 3 P_{\infty}, P_{\infty}+2 P_{0}$ and $3 P_{0}$. Therefore $1, t^{2}, t^{3}$ form a basis of $\lambda$. Since $2 P_{1}+P_{2}, P_{\infty}+P_{1}+P_{3} \in \lambda$, defined by $\bar{H}, C_{1}+C_{2} \in\left|3 C_{1}\right|$, $(t-1)^{2}(t-\alpha)$ and $(t-1)(t-\beta)$ must be written as linear combinations of $1, t^{2}$ and $t^{3}$ with coefficients in $k$. Hence $\alpha=-1 / 2, \beta=-1$, so $P_{0}, P_{1}, P_{2}, P_{3}$ and $P_{\infty}$ are different from each other. Then from the configuration of our basis of $\Lambda$, we obtain (1)-(3) as before.

Let $\mu: \tilde{X} \rightarrow \bar{X}$ and $\tilde{H}$ be as in the theorem and let $X=\Phi_{\mid \tilde{H}}(\tilde{X})$. Then $X$ is a normal hypersurface in $\mathbf{P}^{3}$ of degree 2 or 4 according as $\tilde{H}$ is hyperelliptic or not. It is easy to show that the irreducible curves on $\tilde{X}$ which are contracted by $\Phi_{\mid \tilde{H}}$ are exactly the components of a unique effective anticanonical divisor $\tilde{D}$ of $\tilde{X}$. Moreover, the configuration of $\tilde{D}$ is $2 \tilde{E}_{8}$ or $E 1+A_{1}$ according to our choice of $\bar{D}$. Therefore, for the first part of the theorem it is enough to show the following $L^{2}$.

LEMMA 9. $X$ is a normal quadric surface if and only if $\bar{D}=C_{0}+C_{1}^{\prime}$ and $O_{C_{1}^{\prime}}\left(2 P_{1}\right) \cong$

Proof. Assume $X$ is a normal quadric surface. Then, by Lemma 6, there is a curve $\tilde{C}$ on $\tilde{X}$ such that

$(* *) \tilde{C}$ is nonsingular elliptic, $\tilde{C} \cdot \tilde{H}=2$ and $\operatorname{supp}(\tilde{C}) \cap \operatorname{supp}(\tilde{D})=\varnothing$. Put $C^{\prime}=\mu(\tilde{C})$. Then we have $C^{\prime} \sim m C_{1}$ for some $m \in \mathbf{Z}$ because $C^{\prime}$ is disjoint from $C_{0}$. Set $r_{i}=$ mult $_{P_{i}} C^{\prime}(i=1,2)$. Then we have

$$
\begin{aligned}
& 2=\tilde{C} \cdot \tilde{H}=C^{\prime} \cdot \bar{H}-2 r_{1}-r_{2}=3 m-2 r_{1}-r_{2}, \\
& 0=\tilde{C} \cdot \tilde{D}=C^{\prime} \cdot \bar{D}-r_{1}-r_{2}=m-r_{1}-r_{2} .
\end{aligned}
$$

Hence we get $r_{1}=2, r_{2}=0, m=2$ or $r_{1}=0, r_{2}=1, m=1$. As was shown in the proof of (1)-(3), if such a curve $C^{\prime}$ exists on $\bar{X}$, then $\bar{D}=C_{0}+C_{1}^{\prime}$ with $C_{1}^{\prime}$ a section. We have $C^{\prime} \sim 2 C_{1}^{\prime},\left.C^{\prime}\right|_{C_{1}^{\prime}}=2 P_{1}$ in the former case, and $C^{\prime} \sim C_{1},\left.C^{\prime}\right|_{C_{1}^{\prime}}=P_{2}$ in the latter case. Anyhow we get $O_{C_{1}}\left(2 P_{1}\right) \cong L^{2}$ since $O_{C_{1}}\left(2 P_{1}+P_{2}\right) \cong L^{3}$.

Conversely, let $\bar{D}=C_{0}+C_{1}^{\prime}$ and $O_{C_{1}^{\prime}}\left(2 P_{1}\right) \cong L^{2}$. Then there exists on $\bar{X}$ an irreducible curve $C^{\prime}$ with a double point $P_{1}$ such that $C^{\prime} \sim 2 C_{1}$. The proper transform of $C^{\prime}$ on $\tilde{X}$ satisfies (**) in Lemma 6 and hence $X$ is a normal quadric surface. Q.E.D.

Suppose $X$ is a quartic surface of elliptic ruled type with at least $2 \tilde{E}_{8}$ or $E 1+A_{1}$ as singularities. Let $\pi: \tilde{X} \rightarrow X$ be the minimal resolution of $X$, and let $\tilde{H}$ be the proper transform on $\tilde{X}$ of a general hyperplane section of $X$. Then from Lemmas 2,3 
and the proof of Proposition 2, we have that there exist an elliptic ruled surface $\bar{X}=\mathbf{P}(E) \stackrel{\tilde{\omega}}{\rightarrow} C$ with $C_{0}^{2}=-1$ so we may assume $E=O_{C} \oplus L$ with $\operatorname{deg} L=1, \bar{D} \in$ $\left|-K_{\bar{X}}\right|$ (hence $\bar{D}=C_{0}+C_{1}^{\prime}$ with $C_{1}^{\prime}$ a section or $\bar{D}=2 C_{0}+f_{0}$ with $f_{0}$ a fibre such that $\left.\left.f_{0}\right|_{C_{0}} \cong L\right)$ and a sequence of two points $\left(P_{1}, P_{2}\right)$ on $\operatorname{supp}(\bar{D}) \backslash \operatorname{supp}\left(C_{0}\right)$ such that $\mu: \tilde{X} \rightarrow \bar{X}$ is the blowing-up at $\left(P_{1}, P_{2}\right)$. Put $\bar{H}=\mu(\tilde{H})$. By Lemma $2, P_{1}$ and $P_{2}$ must lie on $\bar{H}$.

LEMMA $10 . \bar{H} \in\left|3 C_{1}-2 P_{1}\right|$.

Proof. Let $\bar{H} \sim m C_{1}$ and let $r_{i}=$ mult $_{P_{1}} \bar{H}(i=1,2)$. Then we have

$$
\begin{aligned}
& 4=\tilde{H}^{2}=\bar{H}^{2}-r_{1}^{2}-r_{2}^{2}=m^{2}-r_{1}^{2}-r_{2}^{2}, \\
& 0=\bar{H} \cdot \bar{D}-r_{1}-r_{2}=m-r_{1}-r_{2} .
\end{aligned}
$$

Hence $m=r_{1}+r_{2}=3$. Since we may assume $r_{1} \geqslant r_{2}$, we get $r_{1}=2$. Q.E.D.

Finally assume $\bar{D}=C_{0}+C_{1}^{\prime}$ and $O_{C_{1}}\left(P_{1}\right) \cong L$, or $\bar{D}=2 C_{0}+f_{0}$ and $P_{1} \in$ $\operatorname{supp}\left(C_{1}\right)$, i.e. $P_{1}$ is the intersection of $f_{0}$ with $C_{1}$. Then, by the basis of $\Lambda=\mid 3 C_{1}-$ $2 P_{1} \mid$ taken in the proof of (1)-(3), we see that the proper transform of a general member of $\Lambda$ on $\tilde{X}$ must have a fixed component, a contradiction. The necessity of condition $(*)$ follows from Lemma 9.

Therefore we complete the proof of Theorem 2 .

Suppose $\bar{D}=C_{0}+C_{1}^{\prime}, O_{C_{1}^{\prime}}\left(P_{1}\right) \not L$ but $O_{C_{1}^{\prime}}\left(2 P_{1}\right) \cong L^{2}$. Let $s_{0}, \ldots, s_{3}$ denote the basis of $\Lambda$ in the first part of this section. Since $f_{1}=f_{1}^{\prime}$ in this case, we get $s_{0} s_{3}=s_{1} s_{2}$. Therefore the induced hypersurface $X$ in $\mathbf{P}^{3}$ is defined by the equation $X_{0} X_{3}=X_{1} X_{2}$ for some homogeneous coordinates $\left(X_{0}, X_{1}, X_{2}, X_{3}\right)$ of $\mathbf{P}^{3}$. Hence, $X$ is a nonsingular quadric surface.

5. Proof of the Corollary. Let $\pi: \tilde{X} \rightarrow X$ denote the minimal resolution of $X$. Looking at the construction of $\tilde{X}$ described in Theorems 1 and 2, but at first without minding condition $(*)$, we can list the possible configuration of curves on $\tilde{X}$ which are disjoint from $\tilde{H}$ (notation is the same as in Theorems 1 and 2), i.e. the possible configuration of the exceptional curves for $\pi$, as fillows:

$\left\{2 \tilde{E}_{7}\right.$, a subgraph of $\left.A_{3}\right\}, \quad\left\{E 1+A_{1}+A_{1}\right.$, a subgraph of $\left.A_{1}+A_{1}\right\}$,

$$
\{E 1+D(3)\}, \quad\{E 1+D(4)\}, \quad\left\{2 \tilde{E}_{8}\right\}, \quad\left\{E 1+A_{1}\right\} .
$$

The possibility of $\left\{E 1+A_{1}+A_{1}, A_{1}, A_{1}\right\}$ arises when we take $\left(P_{1}, P_{2}, P_{3}\right)$ in Theorem 1 so that $\left(P_{1}, P_{2}, P_{3}, P_{4}\right)$ are in the position of (c-3). But in this case the sections of $\tilde{\omega}$ through $P_{1}$ must pass through $P_{3}$. Indeed, suppose $\bar{H} \in \mid 2 C_{1}-P_{1}-P_{2}$ $-P_{3} \mid$ cuts $2 P_{1}$ on $f_{1}$ and $2 P_{3}$ on $f_{2}$. Let $C^{\prime}$ be a section through $P_{1}$ and disjoint from $C_{0}$, and let $P_{3}^{\prime}$ and $P_{0}$ denote the intersections of $f_{2}$ with $C^{\prime}$ and $C_{0}$, respectively. Set $\lambda=\left|2 C_{1}-P_{1}-P_{2}\right|_{\mid f_{2}}$. By

$$
0 \rightarrow O_{\bar{X}}\left(2 C_{1}-f_{1}-f_{2}\right) \rightarrow O_{\bar{X}}\left(2 C_{1}\right) \rightarrow O_{f_{1}+f_{2}}\left(2 C_{1}\right) \rightarrow 0,
$$

we get $\operatorname{dim}\left|2 C_{1}\right|_{\mid f_{1}+f_{2}}=3$, and hence

$$
\operatorname{dim} \lambda \leqslant \operatorname{dim}\left|2 C_{1}-P_{1}-P_{2}\right|_{\mid f_{1}+f_{2}}=1 .
$$


Therefore, from $2 P_{0}, 2 P_{3}, 2 P_{3}^{\prime} \in \lambda$ and $P_{0} \neq P_{3}, P_{3}^{\prime}$, we deduce that $P_{3}=P_{3}^{\prime}$. So condition $(*)$ does not hold in this case.

For the other cases, we can choose $\left(P_{1}, P_{2}, P_{3}\right)$ or $P_{1}$ in Theorems 1 or 2 corresponding to them so that condition $(*)$ is satisfied. This can be proved directly in each case by choosing $P_{i}$ avoiding the finite points which do not satisfy the required conditions. Examples in $\S 6$ also show the existence of quartic surfaces with these types of singularities.

6. Defining equations. Let us find defining equations in suitable homogeneous coordinates of $\mathbf{P}^{3}$ of quartic surfaces $X$ of elliptic ruled type with at least two simple elliptic singularities.

Let $\bar{X}, L, P_{i}(1 \leqslant i \leqslant 4)$ [resp. $i=1,2$ ], etc. be as in Theorem 1 [resp. Theorem 2], where we have seen the correspondence between them and $X$. Set $\Lambda=\mid 2 C_{1}-P_{1}$ $-\cdots-P_{4} \mid$ [resp. $\left.\left|3 C_{1}-2 P_{1}-P_{2}\right|\right]$. To find the defining equation of $X$ it is enough to find a relation of degree 4 of a basis of $\Lambda$.

Case 1. Suppose $X$ has $2 \tilde{E}_{7}$. As before, let $f_{i}(1 \leqslant i \leqslant 8)$ denote the fibre on $\bar{X}$ such that $f_{i}$ passes through $P_{i}(1 \leqslant i \leqslant 4), f_{j}(5 \leqslant i \leqslant 8)$ is disjoint from $P_{i}$ 's, and $f_{5}+f_{6}$, $f_{7}+f_{8} \in\left|\tilde{\omega}^{*} L\right|, f_{5}+f_{6} \neq f_{7}+f_{8}$. Then $A_{0}=2 C_{0}+\Sigma_{i=1}^{4} f_{i}, A_{1}=C_{0}+f_{5}+f_{6}+C_{1}^{\prime}$, $A_{2}=C_{0}+f_{7}+f_{8}+C_{1}^{\prime}$ and $A_{3}=2 C_{1}^{\prime}$ correspond to a basis $s_{0}, s_{1}, s_{2}, s_{3}$ of $\Lambda$. Since $2 A_{0}+2 A_{3}, A_{0}+2 A_{1}+A_{3}, A_{0}+A_{1}+A_{2}+A_{3}, A_{0}+2 A_{2}+A_{3}, 4 A_{1}, 3 A_{1}+A_{2}$, $2 A_{1}+2 A_{2}, A_{1}+3 A_{2}$ and $4 A_{2}$ are greater than $4 C_{0}+4 C_{1}^{\prime}$, and since

$$
\begin{aligned}
\operatorname{dim} & H^{0}\left(\bar{X}, O_{\bar{X}}\left(4 \cdot 2 C_{1}-4 C_{0}-4 C_{1}^{\prime}\right)\right) \\
& =\operatorname{dim} H^{0}\left(\bar{X}, O_{\bar{X}}\left(2\left(f_{1}+f_{2}+f_{3}+f_{4}\right)\right)\right)=8,
\end{aligned}
$$

there exists a nontrivial relation

$$
\begin{aligned}
& a_{1} s_{0}^{2} s_{3}^{2}+s_{0} s_{3}\left(a_{2} s_{1}^{2}+a_{3} s_{1} s_{2}+a_{4} s_{2}^{2}\right) \\
& \quad+a_{5} s_{1}^{4}+a_{6} s_{1}^{3} s_{2}+a_{7} s_{1}^{2} s_{2}^{2}+a_{8} s_{1} s_{2}^{3}+a_{9} s_{2}^{4}=0
\end{aligned}
$$

for some $a_{1}, \ldots, a_{9} \in k$. Hence, if $\left(X_{0}, X_{1}, X_{2}, X_{3}\right)$ are the homogeneous coordinates of $\mathbf{P}^{3}$ corresponding to $\left(s_{0}, s_{1}, s_{2}, s_{3}\right)$, then $X$ is defined by

$$
F\left(X_{0}, X_{1}, X_{2}, X_{3}\right)=a_{1} X_{0}^{2} X_{3}^{2}+X_{0} X_{3} F_{2}\left(X_{1}, X_{2}\right)+F_{4}\left(X_{1}, X_{2}\right)=0,
$$

where $F_{i}$ is a homogeneous polynomial in $X_{1}, X_{2}$ of degree $i(i=2,4)$. Since $X$ has double points at $(1,0,0,0)$ and $(0,0,0,1)$, we have $a_{1} \neq 0$ so we may assume $a_{1}=1$. Moreover, the discriminant of $F$ at $(1,0,0,0)$,

$$
F_{2}^{2}-4 F_{4}=0
$$

is the sum of distinct four lines meeting at $(0,0,1)$ in $\mathbf{P}^{2}$ with homogeneous coordinates $\left(X_{1}, X_{2}, X_{3}\right)$ (cf. the proof of Proposition 1). Therefore, after a suitable change of coordinates, we may assume

(1) $F=\left(X_{0} X_{3}+a X_{1}^{2}+b X_{1} X_{2}+c X_{2}^{2}\right)^{2}+X_{1} X_{2}\left(X_{1}-X_{2}\right)\left(X_{1}-d X_{2}\right)=0$

for some $a, b, c, d \in k, d \neq 0,1$.

Conversely, the quartic surface $X$ defined by an equation of form (1) is normal for any $a, b, c, d \in k$ if $d \neq 0,1$. Moreover, $X$ has singularities of type $\tilde{E}_{7}$ at $(1,0,0,0)$ 
and $(0,0,0,1)$, for the dehomogenizations of (1) at these points are quasi-homogeneous polynomials of weights $(2,4,4)$. Their $j$-invariants are both equal to

$$
2^{8}\left(d^{2}-d+1\right)^{3} / d^{2}(d-1)^{2}
$$

If $\alpha$ is an $(l+1)$ ple root of

$$
c^{2} y^{4}+(2 b c+d) y^{3}+\left(2 a c+b^{2}+d+1\right) y^{2}+(2 a b+1) y+a^{2}=0,
$$

then $X$ also has a rational double point of type $A_{l}$ at $(0,1, \alpha, 0)$. Except for these points $X$ has no singular point.

Case 2. Suppose $X$ has $2 \tilde{E}_{8}$. Let $f_{i}(0 \leqslant i \leqslant 2)$ and $f_{1}^{\prime}$ denote the fibre on $\bar{X}$ such that $f_{i}$ passes through $P_{i}(i=1,2), f_{0} \in\left|\tilde{\omega}^{*} L\right|$ and $f_{1}+f_{1}^{\prime} \in\left|\tilde{\omega}^{*} L^{2}\right|$. Let $s_{0}, s_{1}, s_{2}, s_{3}$ be the basis of $\Lambda$ corresponding to $3 C_{0}+2 f_{1}+f_{2}, 2 C_{0}+f_{1}+f_{1}^{\prime}+C_{1}^{\prime}, C_{0}+f_{0}+$ $2 C_{1}^{\prime}$ and $3 C_{1}^{\prime}$, respectively. Then, in a similar way as in the above case, we have a nontrivial relation

$$
a_{1} s_{0}^{2} s_{3}^{2}+a_{2} s_{0} s_{1} s_{2} s_{3}+a_{3} s_{1}^{3} s_{3}+a_{4} s_{0} s_{2}^{3}+a_{5} s_{1}^{2} s_{2}^{2}=0
$$

for some $a_{1}, \ldots, a_{5} \in k$. So $X$ is defined by

$$
F=a_{1} X_{0}^{2} X_{3}^{2}+a_{2} X_{0} X_{1} X_{2} X_{3}+a_{3} X_{1}^{3} X_{3}+a_{4} X_{0} X_{2}^{3}+a_{5} X_{1}^{2} X_{2}^{2}=0 .
$$

Moreover, since $X$ has double points at $(1,0,0,0)$ and $(0,0,0,1)$, and since the discriminant of $F$ at $(0,0,0,1)$,

$$
\left(a_{2} X_{1} X_{2}+a_{4} X_{2}^{3}\right)^{2}-4 a_{1}\left(a_{3} X_{1}^{3}+a_{5} X_{1}^{2} X_{2}^{2}\right)=0,
$$

is reduced, we may assume $a_{1}=a_{3}=a_{4}=1$, so

$$
F=X_{0}^{2} X_{3}^{2}+X_{1}^{3} X_{3}+X_{0} X_{2}^{3}+a X_{0} X_{1} X_{2} X_{3}+b X_{1}^{2} X_{2}^{2}=0
$$

for some $a, b \in k$.

Conversely, any quartic surface defined by an equation of form (2) has, if it is normal, singularities of type $\tilde{E}_{8}$ at $(1,0,0,0)$ and $(0,0,0,1)$. This surface is normal if and only if

$$
\Delta=a^{4} b-a^{3}-8 a^{2} b^{2}+36 a b+16 b^{3}-27 \neq 0 .
$$

In this case, the $j$-invariants of the two singularities are both equal to

$$
\left(\left(a^{2}-4 b\right)^{2}+24 a\right)^{3} / \Delta .
$$

For quartic surfaces of elliptic ruled type with a singularity of $p_{g}=2$, we give only typical examples of their defining equations:

(4) $E 1+D(3):\left(X_{0} X_{1}+X_{2}^{2}+X_{3}^{2}\right)^{2}+X_{1}^{4}+X_{1} X_{3}^{3}=0$,

(5) $E 1+D(4):\left(X_{0} X_{1}+X_{2}^{2}\right)^{2}+X_{1}^{4}+X_{1} X_{3}^{3}=0$,

(6) $E 1+A_{1}: X_{0}^{2} X_{1}^{2}+X_{0} X_{2}^{3}-3 X_{0} X_{1} X_{2} X_{3}+16 X_{1}^{4}+2 X_{1} X_{3}^{3}-\frac{3}{4} X_{2}^{2} X_{3}^{2}=0$. 
All of these surfaces have unique singularity at $(1,0,0,0)$. On the other hand,

$$
\left(X_{0} X_{1}+X_{2}^{2}+\sqrt{-1} X_{3}^{2}\right)^{2}+X_{1}^{4}+X_{3}^{4}=0
$$

has a singularity of type $E 1+A_{1}+A_{1}$ at $(1,0,0,0)$ and of type $A_{1}$ at $(0,0,0,1)$.

Equations (3), (4) and (6) were pointed out to the author by K. Watanabe, Y. Koyama and M. Kato, respectively.

\section{REFERENCES}

I. F. Hidaka and K. Watanabe, Normal Gorenstein surfaces with ample anticanonical divisor, Tokyo J. Math. 4 (1981), 319-330.

2. H. Laufer, On minimally elliptic singularities, Amer. J. Math. 99 (1977), 1259-1295.

3. Y. Umezu, On normal projective surfaces with trivial dualizing sheaf, Tokyo J. Math. 4 (1981), 343-354.

4. Stephen S.-T. Yau, On maximally elliptic singularities, Trans. Amer. Math. Soc. 257 (1980), 269-329. 5. (1979), 761-812.

6. Gorenstein singularities with geometric genus equal to two, Amer. J. Math. 101 (1979), $813-854$

Department of Mathematics, faculty of Sciences, Tokyo Metropolitan University, Fukazawa, Setagaya-Ku, TOKyo 158 